\title{
Can obesity in early childhood be influenced by lifestyle interventions during pregnancy? A systematic review of the literature
}

\author{
K. Dalrymple ${ }^{1}$, J. Martyni-Orenowicz ${ }^{1}$, M. O’Keeffe ${ }^{2}$ and L. Poston ${ }^{1}$ \\ ${ }^{1}$ Division of Women's Health, King's College London, St Thomas' Hospital, London, SE1 7EH, UK and \\ ${ }^{2}$ Division of Diabetes and Nutritional Sciences, King's College London, London, SE1 9NH, UK
}

This abstract was awarded the student prize for best poster original communication.

Childhood obesity is rapidly becoming a global pandemic, in 2015 more than 41 million children $<5$ years were affected by overweight or obesity ${ }^{(1)}$. In the UK, over a fifth of children aged 4-5 years are classified as overweight or obese ${ }^{(2)}$. There is an increasingly convincing body of evidence that obesity in children may have its origins in early life, specifically adverse exposures during in-utero development ${ }^{(3)}$. Cohort studies have highlighted the association between childhood obesity and increased maternal body mass index $(\mathrm{BMI})^{(4,5)}$ and excessive gestational weight gain ${ }^{(5)}$. Furthermore, observational studies suggest that manipulation of maternal diet and/or physical activity in the antenatal period has the potential to influence offspring growth and development ${ }^{(6)}$ and this has been shown in experimental animals and their offspring ${ }^{(7)}$. The maternal population is therefore a target for prevention of childhood obesity, and this has led to a number of randomised controlled trials focusing on lifestyle in pregnancy. However, any lasting causal effect on childhood obesity from these trials is currently unknown.

The objective of this systematic review was to determine whether antenatal lifestyle interventions in pregnant women, aimed at modifying diet and/or physical activity, lead to a reduction in measures of offspring obesity in early childhood.

A systematic review of the literature was completed from $1^{\text {st }}$ January $1990-31^{\text {st }}$ March 2017 in MEDLINE, Embase, and CENTRAL for antenatal interventions with subsequent offspring follow-up publications. A hand search of reference lists and cited articles of relevant reports and review articles was also completed. Electronic searches identified 3361 titles and a further three trials were identified through hand searches. Of these 145 duplicates were removed, 32 abstracts were eligible for full-text review, KD and JMO independently screened the relevant articles, extracted the data and assessed risk of bias.

Seven antenatal lifestyle interventions with offspring follow-up data were identified. Four trials included women from all BMI categories and three trials included obese women only. The antenatal interventions were heterogeneous in terms of intervention design, intensity and outcome measures. For the seven offspring follow-up publications children aged 6 months-7 years, measures of obesity in the offspring $(n=1689)$ included, weight, BMI, weight-for-length z-scores, skinfold thicknesses and circumferences. Two studies, focusing on obese women only, reported reduced measures of adiposity from $6-12$ months $(\mathrm{n}=787)$. The remaining five studies, two from infancy $(n=500)$ and three $(n=402)$ from early childhood found no effect on measures of obesity.

For the offspring follow-up studies, there was heterogeneity in methods and variations in reported outcomes and studies were limited due to high rates of attrition. Measures of obesity up to 12 months of age have been shown to be reduced by lifestyle interventions during pregnancy in obese women, however we are unable to draw a conclusion on the influence antenatal interventions have on measures of obesity in early childhood.

1. UNICEF, WHO, World Bank (2015) Levels and trends in child malnutrition. New York: UNICEF, Geneva: WHO, Washington DC: World Bank.

2. Health \& Social Care (2016) National Child Measurement Programme 2015/2016. London: NHS

3. Godfrey K, Reynolds R, Prescott S et al. (2016) Lancet Diabetes Endocrinol 5, 51-64.

4. Castillo H, Santos I, Matijasevich A (2015) Maternal \& Child Nutrition 11, 606-617.

5. Yu Z, Han S, Zhu J et al. (2013) PloS one 8, e61627.

6. Nelson SM, Matthews P, Poston L (2010) Human Reprod Update 16, 225-75

7. Zambrano E, Martínez-Samayoa PM, Rodríguez-González GL et al. (2010) J Physiol 588, 1791-1799. 